\title{
Pup age and aggressive behavior in lactating rats
}

M. Giovenardi'

A.R. Consiglio ${ }^{2}$,

H.M.T. Barros ${ }^{3}$ and

A.B. Lucion ${ }^{1}$

\author{
Departamentos de ${ }^{1}$ Fisiologia and ${ }^{2}$ Biofísica, \\ Universidade Federal do Rio Grande do Sul, Porto Alegre, RS, Brasil \\ ${ }^{3}$ Divisão de Farmacologia, Fundação Faculdade Federal de Ciências Médicas, \\ Porto Alegre, RS, Brasil
}

\section{Correspondence \\ A.B. Lucion \\ Departamento de Fisiologia Instituto de Ciências Básicas da Saúde, UFRGS \\ Rua Sarmento Leite, 500 \\ 90050-170 Porto Alegre, RS Brasil \\ E-mail: alucion@ vortex.ufrgs.br \\ Research supported by FAPERGS, CNPq, FINEP and CAPES.}

Received O ctober 21, 1999 Accepted June 21, 2000

\section{Abstract}

High levels of aggressive behaviors against intruders in the nest area are displayed by female rats during the first 10 days after delivery, declining thereafter to very low levels, even though lactation continues. Cross-fostering experiments were undertaken to test the hypothesis that pup age may affect aggression in lactating rats. The behavior of females on the 8th day after delivery when raising fostered 8day-old pups was compared to that of females on the 8th postpartum day raising older pups ( 18 days old) for the last 5 days, and females on the 18th day after delivery raising fostered 18-day-old pups were compared to females in the same postpartum period nursing younger pups ( 8 days of age at the time of the maternal aggression test) for 5 days. Pup retrieval activity and plasma prolactin level were also analyzed. Females on the 8th postpartum day nursing 18-day-old pups were less aggressive than females in the same postpartum period, but with 8-day-old pups. Likewise, females on the 18th postpartum day nursing younger pups were more aggressive and presented higher levels of prolactin than females nursing older pups. Thus, pup development can alter the natural decline of maternal aggressive behavior.

\section{Introduction}

Maternal behavior in mammals is one of most stable and highly motivated speciesspecific behaviors $(1,2)$. The aggressive behavior of lactating females is part of the parental care (3-5), which includes several other protective activities (6). While much work has focused on the study of maternal caretaker behaviors, less is known about the nature and etiology of aggression during the postpartum period.

The marked hormonal changes at the end of pregnancy and during the early postpar-

\section{Key words}

- Maternal aggression

- Mother-infant interaction

- Cross-fostering

- Prolactin tum period have been considered to be the endogenous substrates that may influence the onset of maternal behaviors (for a review, see 7). However, the continuous exposure of virgin and ovariectomized female rats without hormonal treatment, as well as males, to pups can elicit maternal behavior $(8,9)$.

On the other hand, the onset of maternal aggressive behavior in rodents seems to require both the hormonal changes occurring at the end of pregnancy and the presence of the pups (10-12). Previous work showed that prolactin is related to the emergence of post- 
partum aggression in mice and hamsters $(13,14)$. In rats, gonadal hormones influence the onset of maternal aggression $(10,15,16)$. Indeed, in nonpregnant ovariectomized rats treated with estradiol and progesterone $(17,18)$ as well as in lactating female rats (19), the presence of the pups and their somatosensory stimulation on the mother are necessary to induce aggressive behavior. Moreover, the number of pups increases the propensity of the mother to attack the intruder (5). In mice, suckling can induce aggressive behavior in virgin females $(11,20)$; in rats, however, suckling alone did not provide sufficient stimulation to elicit maternal aggression $(19,21)$.

During the first 10 days after delivery, female rats vigorously attack intruders in the nest area, and thereafter this behavior declines to very low levels, even though lactation continues $(22,23)$. The factors that elicit it appear to be different from those that maintain aggressive behavior during the first 10 days after delivery (for a review, see 7). The natural decline of maternal aggressive behavior after the 10th postpartum day is less understood than its onset. Previous work (24) addressed this issue and showed no relationship between the physical development of the pups and the decline of aggressiveness.

The aim of the present research was to study the effects of age, and therefore of developmental stage and behavior of the pups, on the aggressive behavior of lactating female rats. The presence of the pups is necessary for the aggressive behavior of the dam, since their separation decreases this behavior (19). As the pups grow old, they depend less on maternal care in terms of feeding, although suckling per se does not seem to elicit maternal aggression (21) or protection. On the other hand, dams are less stimulated by older pups than younger ones due to their increased independence. Crossfostering experiments were undertaken to test the hypothesis that pup age could affect maternal aggression. We planned to test the aggressive behavior of the lactating female 5 days after the cross-fostering procedure. We expected that the longer period of contact between the mother and the fostered pups, as compared to the study of Albert and Walsh (24), that involved only a 2-day interval, would affect maternal aggression. The behavior of females was tested on the 8th postpartum day with 18-day-old pups. Likewise, females on the 18th day after delivery with 8-day-old pups were tested against a male intruder. We hypothesized that the interaction for 5 days with younger pups would increase the aggressive behavior of females on the 18th postpartum day and, conversely, the interaction with older pups would decrease the aggressiveness of females on the 8th day after delivery. We chose to test the aggressive behavior on the 8th and on the 18th day in order to leave the fostered pups with the dams during a naturally high (from the 3rd to the 8th day) or low (from the 13th to the 18th postpartum day) aggressiveness period. In addition, we also tested the effects of this treatment on the hormonal levels of the dams. Although previous work (25) showed no significant reduction in maternal aggressive behavior (only a tendency) after hypophysectomy, which would discount a possibly role of prolactin on this behavior, we planned to measure plasma prolactin levels during a high- and a low-aggression period. Since plasma prolactin levels decline in the second half of the postpartum period $(26,27)$, we aimed to confirm whether younger pups (8 days old) would affect the levels of dams on the 18th day after delivery.

\section{Material and Methods}

\section{Animals and treatments}

Lactating female Wistar rats from the stock of the Federal University of Rio Grande do Sul weighing $240 \pm 20 \mathrm{~g}$ were used in the experiment. The animals were maintained 
on a light/dark (12/12 h) cycle with lights off at 16:00 $\mathrm{h}$ and the behavior recordings (aggressive and retrieval) were performed during the dark phase from 16:00 to 18:30 h. The temperature was kept at $22^{\circ} \mathrm{C}$ and the animals had free access to food and water.

Females were tested against a male intruder on the 8th and 18th day after delivery. In all groups, pups were cross-fostered 5 days before the behavioral test (3rd and 13th postpartum day). The number of pups per dam was set at 7 .

Females were divided into 4 groups: group F8+P8 $(\mathrm{N}=14)$, females tested on the 8th day after delivery raising 8-day-old pups; group F8+P18 $(\mathrm{N}=13)$, females tested on the 8 th day after delivery raising 18-day-old pups; group $\mathrm{F} 18+\mathrm{P} 18(\mathrm{~N}=10)$, females tested on the 18th day after delivery raising 18-day-old pups, and group F18+P8 $(\mathrm{N}=$ 19 ), females tested on the 18th day after delivery raising 8 -day-old pups. The crossfostering procedures adopted were the same as in previous studies $(3,28)$. By observing the dams and pups for some hours after cross-fostering and on the next day, we confirmed that the females did not reject them. All cross-fostered pups were grouped in the nest and they did not show any evident sign of physical suffering.

\section{Behavior recording}

Females were first mated with males and, after being considered pregnant by visual and manual inspection, they were housed individually in $50 \times 45 \times 25-\mathrm{cm}$ Plexiglas home-cages with wood shavings for bedding and nest building. On the 8 th or the 18 th day after delivery, the behaviors of the female in the presence of an intruder male were videotaped for 10 min beginning immediately after placing the intruder into the home-cage where the female was housed with her crossfostered pups. The intruders were young males smaller than the females and were used only once. In this study and in other studies from this laboratory $(3,29,30)$ using the same rat strain, no pup was attacked by the intruder. The behaviors of the females towards the intruder recorded during the maternal aggression tests have been described elsewhere $(3,31)$, and can be briefly summarized as sniffing, lateral attack, frontal attack and biting.

In order to avoid a possible interference of aggressive behavior with the retrieval activity, we measured it $24 \mathrm{~h}$ after the maternal aggression test. In the retrieval test, the female was removed from the home-cage and the pups were distributed around in the cage. The latency (s) to retrieve the 1st and the 7th pup was recorded only in the groups in which pups were 9 days old and raised by dams on the 9th or 19th postpartum day (groups F8+P8 and $\mathrm{F} 18+\mathrm{P} 8$ ).

\section{Prolactin radioimmunoassay}

Immediately after the retrieval test, on the 9 th and 19th day after delivery and about 30 min after the beginning of the dark phase of the cycle, females of all groups were killed by decapitation and blood was collected from the trunk. Samples were centrifuged for $10 \mathrm{~min}$ at $3000 \mathrm{rpm}$; the plasma was separated and stored frozen and the concentration of prolactin was determined by radioimmunoassay according to the method described by Niswender et al. (32). Anti-rat PRL-S9 was the specific prolactin antibody used and PRL-RP3 was the standard preparation adopted. The precipitation antibody (2nd antibody) was produced in the laboratory of Dr. Celso R. Franci (Faculty of Medicine of Ribeirão Preto, University of São Paulo, Ribeirão Preto, SP, Brazil), and all assays were performed in this laboratory.

\section{Statistical analysis}

In order to test the effects of pup age on the behavior of the dams, comparisons were performed between dams during the same 
postpartum period. The mean ( \pm SEM) frequency of behaviors during the 10-min recording sessions was compared between the 2 groups of dams on the 8th (F8+P8 compared to F8+P18) and 18th postpartum day (F18+P18 compared to F18+P8) using the Student $t$-test. The mean $( \pm \mathrm{SEM})$ concentration of plasma prolactin was also compared between the same groups using the Student $t$ test. The median latency (s) to retrieve the pups was compared between groups F $8+\mathrm{P} 8$ and F18+P8 using the Mann-Whitney Utest. In all comparisons, $\mathrm{P}<0.05$ was considered statistically significant.

Figure 1 - Mean $( \pm$ SEM) frequency of sniffing, lateral attack, frontal attack and biting the intruder male by the lactating female with cross-fostered pups during $10-$ min recording sessions. F8+P8 (female on the 8th postpartum day raising 8-day-old pups); F8+P18 (female on the 8th postpartum day raising 18day-old pups); F18+P18 (female on the 18th postpartum day raising 18-day-old pups); F18+P8 (female on the 18th postpartum day raising 8-day-old pups). $\mathrm{N}=$ Number of animals in each group. $* \mathrm{P}<0.05$, F8+P18 compared to group F8+P8; $+\mathrm{P}<0.05$, F18+P8 compared to group F18+P18 (Student t-test).

Figure 2 - Mean ( \pm SEM) level of plasma prolactin of lactating females on the 9th and 19th postpartum day (one day after the aggressive behavior test) with cross-fostered pups. See Figure 1 for group identification. $\mathrm{N}=$ Number of animals in each group. $* \mathrm{P}<0.05$ compared to group F18+P18 (Student t-test).
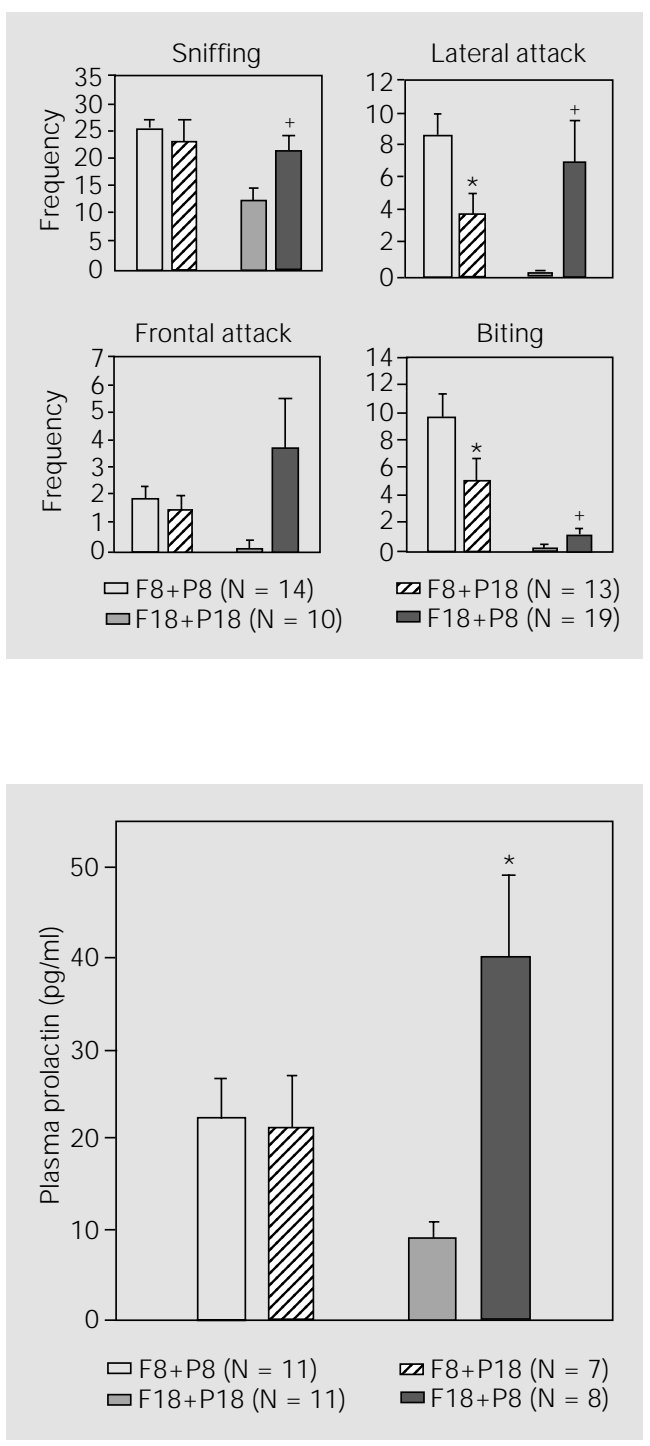

\section{Results}

Figure 1 shows that on the 8th postpartum day, the frequency of lateral attack and biting in group F8+P18 was lower than in group F8+P8. On the 18th postpartum day, the frequency of sniffing, lateral attack and biting was higher in group $\mathrm{F} 18+\mathrm{P} 8$ than in group $\mathrm{F} 18+\mathrm{P} 18$.

Both the latencies to retrieve the first and the last pup of group F18+P8 (12 (4/37) and $50(40 / 152)$ median and interquartile range, respectively) were significantly different from group F8+P8 (71.5 (14/900) and 235.5 (95/900), respectively). However, comparisons of the effect of pup age on dam behavior could not be performed because the 19 day-old pups (age at the day of the retrieval test) were already very mobile and therefore the retrieval test would be meaningless. Nevertheless, results showed that females on the 19th day after delivery were able to retrieve the pups very quickly.

Figure 2 shows that on the 19th postpartum day the plasma prolactin concentration in group $\mathrm{F} 18+\mathrm{P} 8(\mathrm{~N}=8)$ was higher than in group $\mathrm{F} 18+\mathrm{P} 18(\mathrm{~N}=11)$. On the 9 th postpartum day (day of the test), the 2 groups of mothers $(\mathrm{F} 8+\mathrm{P} 8, \mathrm{~N}=11$ and $\mathrm{F} 8+\mathrm{P} 18, \mathrm{~N}=7)$ did not present any difference.

\section{Discussion}

Results showed that females on the 8th postpartum day which had been nursing older pups for the previous 5 days were less aggressive than females in the same postpartum period, but with 8-day-old pups. Likewise, females on the 18th postpartum day nursing younger pups ( 8 days old at the time of the maternal aggression test) for the previous 5 days were more aggressive than females nursing 18 -day-old pups. On the other hand, plasma prolactin concentration in females on the 19th postpartum day nursing younger pups ( 9 days old at the time of the test) for 6 days was higher than in females 
during the same lactation period, but raising 19-day-old pups.

The results of the present study suggest that pup age may affect the maternal aggressive behavior. Younger pups fostered for 5 days by females that are in a naturally low aggressive period (after postpartum day 13) may increase the aggressive behavior of the mother, and older pups may decrease maternal aggression.

Previous work (24) showed no difference in maternal aggressive behavior of LongEvans lactating females on the 11th and 19th postpartum day nursing 4-day-old pups. However, in the previous study, 2-day-old pups were cross-fostered and the aggressive behavior test was performed 2 days later, while in the present study they remained 5 days with the cross-fostered dam. We may speculate that the duration of fostering ( 5 days in our experiment), and therefore its cumulative effect, could be an important variable to change the behavior of the dams.

The behavior of the pups could explain the increased aggressiveness. Pups from 3 to 8 days of age remain in the nest most of the time and are more dependent on the mother. At 13 to 18 days of age, pups have already opened their eyes and show spontaneous locomotion in the environment (33-35). It may be suggested that the degree of independence of the pups from the mother may be the cause of the reversed effects on maternal aggression. Maternal aggressive behavior is an interaction between the female and a male intruder, but seems to depend on the motherinfant relationship.

The increased level of plasma prolactin in females on the 19th postpartum day raising younger pups ( 9 days old) during the previous 6 days compared to females during the same postpartum period raising older pups could be explained by the higher frequency of nipple stimulation. However, females on the 9th postpartum day raising older pups (19 days old) during the previous 6 days showed no difference compared to females during the same postpartum period raising younger pups. Results also showed that the increased plasma prolactin level observed in females 19 days postpartum, raising 9-day-old pups, was correlated with an increased aggressive behavior. On the other hand, females 8 days postpartum raising 18 day-old pups showed a decrement of maternal aggression, but no change in plasma prolactin measured one day after the aggressive behavior test. Previous studies on rats showed that during the first days after delivery dams spent most of their time nursing the pups, while, during the third postpartum week, there was a marked reduction in time spent in the litter chamber $(36,37)$. Plasma prolactin levels were lower during the second half of the lactating period than during the first $(26,27)$. We may infer that the increased plasma prolactin levels 19 days postpartum in dams raising 9-day-old pups may be due to increased nursing activity, although we did not measure pup body weight.

We may conclude that the higher the mother-infant physical contact, the greater the aggressiveness of the dam. The increased survival independence of the pups in terms of feeding and environmental exploration may decrease the mother's protective behaviors.

\section{Acknowledgments}

We are grateful to Dr. Celso R. Franci and Sonia A. Zanon for the radioimmunoassay. 


\section{References}

1. Clutton-Brock TH (1991). The Evolution of Parental Care. Princeton University Press, New J ersey.

2. Stern J M (1997). Offspring-induced nurturance: animal-human parallels. Developmental Psychobiology, 31: 19-37.

3. Lucion $A B \&$ De Almeida RMM (1996). On the dual nature of maternal aggression in rats. Aggressive Behavior, 22: 365373.

4. Erskine MS, Denenberg VH \& Goldman BD (1978). Aggression in the lactating rat: effects of intruder age and test arena. Behavioral Biology, 23: 52-66.

5. Mos J , Olivier B, Oorschot RV, Aken HV \& Zethof T (1989). Experimental and ethological aspects of maternal aggression in rats: Five years of observation. In: Blanchard RJ, Brain PF, Blanchard C\& Parmigiani S (Editors), Ethoexperimental Approaches to Study of Behavior. Vol. 1. Kluwer Academy Publishers, Dordrecht, 385-398.

6. Stern J M \& J ohnson SK (1990). Ventral somatosensory determinants of nursing behavior in Norway rats. I. Effects of variations in the quality and quantity of pup stimuli. Physiology and Behavior, 47: 9931011.

7. Numan M (1994). Maternal behavior. In: Knobil E \& Neill J D (Editors), The Physiology of Reproduction. Raven Press, New York, 221-302.

8. Rosenblatt J S, Hazelwood S \& Poole J (1996). Maternal behavior in male rats: Effects of medial preoptic area lesion and presence of maternal aggression. Hormones and Behavior, 30: 201-215.

9. Rosenblatt J S (1967). Nonhormonal basis of maternal behavior in the rat. Science, 156: 1512-1514.

10. Mayer AD \& Rosenblatt J S (1987). Hormonal factors influence the onset of maternal aggression in laboratory rats. Hormones and Behavior, 21: 253-267.

11. Svare B \& Gandelman R (1976). Suckling stimulation induces aggression in virgin female mice. Nature, 260: 606-608.

12. Gandelman R \& Simon NG (1980). Postpartum fighting in the rat nipple development and the presence of young. Behavioral and Neural Biology, 28: 350-360.

13. Svare B, Mann M, Broida J \& Michael SD (1982). Maternal aggression exhibited by hypophysectomized parturient mice. Hormones and Behavior, 16: 455-461.

14. Wise DA (1974). Aggression in the female golden hamster: effects of reproductive state and social isolation. Hor- mones and Behavior, 5: 235-250.

15. Albert DJ , J onik RH \& Walsh ML (1992). Interaction of estradiol, testosterone, and progesterone in the modulation of hormone-dependent aggression in the female rat. Physiology and Behavior, 52: 773-779.

16. Albert DJ \& Walsh ML (1995). Aggression is attenuated by ovariectomy in pregnant female rats given progesterone and estradiol replacement to maintain pregnancy. Physiology and Behavior, 57: 1035-1038.

17. Mayer AD, Monroy MA \& Rosenblatt J S (1990). Prolonged estrogen-progesterone treatment of nonpregnant ovariectomized rats: factors stimulating home-cage and maternal aggression and short-latency maternal behavior. Hormones and Behavior, 24: 342-364.

18. Mayer AD \& Rosenblatt J S (1993). Persistent effects on maternal aggression of pregnancy but not of estrogen/progesterone treatment of nonpregnant ovariectomized rats revealed when initiation of maternal behavior is delayed. Hormones and Behavior, 27: 132-155.

19. Stern J M \& Kolunie J M (1993). Maternal aggression of rats is impaired by cutaneous anesthesia of the ventral trunk, but not by nipple removal. Physiology and Behavior, 54: 861-868.

20. Svare B, Mann M \& Samuels SO (1980). Mice-suckling stimulation but not lactation is important for matemal aggression. Behavioral and Neural Biology, 29: 453462.

21. Factor EM, Mayer AD \& Rosenblatt J S (1992). Preventing suckling-induced release of oxytocin does not inhibit maternal aggression in lactating rats. Annals of the New York Academy of Sciences, 652: 423-424.

22. Flannelly KJ \& Flannelly L (1987). Time course of postpartum aggression in rats (Rattus norvegicus). J ournal of Comparative Psychology, 101: 101-103.

23. Mayer $A D$, Reisbick $S$, Siegel $H I \&$ Rosenblatt J S (1987). Maternal aggression in rats: changes over pregnancy and lactation in a Sprague-Dawley strain. Aggressive Behavior, 13: 29-43.

24. Albert DJ \& Walsh ML (1995). Aggression in the lactating female rat: the normal decline is not dependent on the physical development of the pups. Physiology and Behavior, 58: 477-481.

25. Erskine MS, Barfield RJ \& Goldman BD (1980). Postpartum aggression in rats: effects of hypophysectomy. J ournal of
Comparative and Physiological Psychology, 94: 484-494.

26. Tucker HA (1991). Lactation and its hormonal control. In: Knobil E \& Neill J D (Editors), The Physiology of Reproduction. Raven Press, New York, 1065-1089.

27. Amenomori $Y$, Chen $C L \&$ Meites J (1970). Serum prolactin levels in rats during reproductive states. Endocrinology, 86: 506-510.

28. Lucion $A B$, De Almeida RMM \& De Marques AAB (1994). Influence of the mother on development of aggressive behavior in male rats. Physiology and Behavior, 55: 685-689.

29. Consiglio AR \& Lucion AB (1996). Lesion of hypothalamic paraventricular nucleus and maternal aggressive behavior in rats. Physiology and Behavior, 59: 591-596.

30. De Almeida RMM \& Lucion AB (1994). Effects of intracerebroventricular administration of 5-HT receptor agonists on the maternal aggression of rats. European J ournal of Pharmacology, 264: 445-448.

31. Giovenardi M, Padoin MJ , Cadore LP \& Lucion AB (1998). Hypothalamic paraventricular nucleus modulates maternal aggression in rats: effects of ibotenic acid lesion and oxytocin antisense. Physiology and Behavior, 63: 351-359.

32. Niswender GD, Chen $C L$, Midgley $A R$, Meites J \& Ellis S (1969). Radioimmunoassay for rat prolactin. Proceedings of the Society for Experimental Biology and Medicine, 130: 793-797.

33. Allin JT \& Banks EM (1972). Functional aspects of ultrasound production by infant albino rats. Animal Behavior, 20: 175185.

34. Deviterne D, Desor D \& Krafft B (1990). Maternal behavior variations and adaptations, and pup development within litters of various sizes in Wistar rats. Developmental Psychobiology, 23: 349-360.

35. Lucion AB, Muradas A \& Teixeira L (1997). Effects of neonatal stimulation on behavior development in rats. International Society for Developmental Psychobiology Annual Meeting Abstract, New Orleans, LA, USA, 23: 1081.

36. Grota LJ \& Ader R (1969). Continuous recording of matemal behavior in Rattus norvegicus. Animal Behaviour, 17: 722729 .

37. Grota LJ \& Ader R (1974). Behavior of lactating rats in dual-chambered maternity cage. Hormones and Behavior, 5: 275282. 